Original article

\title{
Family history of alcohol use disorder is associated with brain structural and functional changes in healthy first-degree relatives
}

\author{
Irina Filippi ${ }^{\mathrm{a}}$, Nicolas Hoertel ${ }^{\mathrm{b}, \mathrm{c}, \mathrm{f}, 1}$, Eric Artiges ${ }^{\mathrm{a}, \mathrm{g}, 1}$, Guillaume Airagnes ${ }^{\mathrm{b}, \mathrm{c}, \mathrm{d}, \mathrm{e}}$, \\ Christophe Guérin-Langlois ${ }^{\mathrm{b}, c, 1}$, Anne-Sophie Seigneurie ${ }^{\mathrm{b}, \mathrm{c}}$, Pauline Frère ${ }^{\mathrm{a}}$, \\ Manon Dubol $^{\mathrm{a}}$, François Guillon ${ }^{\mathrm{b}, \mathrm{c}}$, Hervé Lemaître ${ }^{\mathrm{a}}$, Mehdi Rahim ${ }^{\mathrm{h}}$, \\ Jean-Luc Martinot ${ }^{\mathrm{a}, *, 2}$, Frédéric Limosin ${ }^{\mathrm{b}, c, e, 2}$
}

\author{
a INSERM Unit 1000 "Neuroimaging \& Psychiatry", University Paris Sud, University Paris Saclay, University Paris Descartes, Digiteo-labs, Gif-sur-Yvette, France \\ ${ }^{\mathrm{b}}$ Department of Psychiatry and Addictology, Hôpitaux Universitaires Paris Ouest, Assistance Publique-Hôpitaux de Paris, Paris, France \\ c Paris Descartes University, Pôles de recherche et d'enseignement supérieur Sorbonne Paris Cité, Paris, France \\ d INSERM UMS 011, Population-based Epidemiological Cohorts, Villejuif, France \\ e Inserm UMR 1168, VIMA, Villejuif, France \\ IINSERM UMR 894, Psychiatry and Neurosciences Center, Paris, France \\ ${ }^{g}$ GH Nord Essonne, Department of Psychiatry 91G16, Orsay Hospital, Orsay, France \\ ${ }^{\text {h } C E A, ~ D S V, ~ I 2 B M, ~ N e u r o s p i n, ~ G i f-s u r-Y v e t t e, ~ F r a n c e ~}$
}

\section{A R T I C L E I N F O}

\section{Article history:}

Received 5 February 2019

Received in revised form 5 August 2019

Accepted 26 August 2019

Available online 24 September 2019

\section{Keywords:}

Alcohol use disorder

Childhood Trauma Questionnaire

Monetary Incentive Delay task

Relatives

Voxel-Based Morphometry

Vulnerability

\begin{abstract}
A B S T R A C T
Background: Neuroimaging studies of vulnerability to Alcohol Use Disorder (AUD) have identified structural and functional variations which might reflect inheritable features in alcohol-naïve relatives of AUD individuals ( $\mathrm{FH}+$ ) compared to controls having no such family history (FH-). However, prior research did not simultaneously account for childhood maltreatment, any clinically significant disorder and maternal AUD. Therefore, we mainly aimed to investigate the brain structure and reward-related neural activations (fMRI), using whole-brain analysis in $\mathrm{FH}+$ young adults with no prevalent confounders. Methods: $46 \mathrm{FH}+$ and $45 \mathrm{FH}$ - male and female participants had no severe childhood maltreatment exposure, neither any psychiatric disorder or AUD, nor a prenatal exposure to maternal AUD. We used a 3 T MRI coupled with a whole brain voxel-based method to compare between groups the grey matter volumes and activations in response to big versus small wins during a Monetary Incentive Delay task. The Childhood Trauma Questionnaire score was used as confounding variable in the analyses to account for the remaining variance between groups.

Results: Compared to FH- controls, FH+ participants had smaller grey matter volumes in the frontal and cingulate regions as well as in the bilateral nucleus accumbens and right insula. The FH+ participants' fMRI datasets denoted a blunted activation in the middle cingulum with respect to FH- controls' during the processing of reward magnitude, and a greater activation in the anterior cingulum in response to anticipation of a small win.

Conclusions: Family history of alcohol use disorder is linked to structural and functional variations including brain regions involved in reward processes.
\end{abstract}

(C) 2019 Published by Elsevier Masson SAS.

\section{Introduction}

Alcohol use disorder (AUD) is a leading cause of mortality, morbidity and disability worldwide [1]. AUD has been attributed to

\footnotetext{
* Corresponding author at: INSERM Unit 1000 Neuroimaging \& Psychiatry, Digiteo-labs, bâtiment 660, rue Noetzlin, 91190, Gif-sur-Yvette, France.

E-mail address: jean-luc.martinot@inserm.fr (J.-L. Martinot).

1 Authors contributed equally.

2 Authors contributed equally.
}

genetic predisposition for up to 40 to $60 \%$ [2,3], hence this risk in first-degree relatives of AUD individuals is much greater than in the general population [4]. Neuroimaging studies conducted among AUD individuals discussed inherent pre-existing variations entangled with the neurotoxic effects of alcohol use per se [5]. Hence, investigations in adolescents from AUD families provided evidence of structural [6-10] and functional [11-14] variations possibly reflecting vulnerability to AUD. In addition, there are prevalent confounders which may affect brain maturation such as childhood maltreatment, which is associated with psychopathology and risk of substance abuse $[15,16]$. Therefore, the previous 
findings need to be re-evaluated to consider the prepotent influence of the maltreatment on structural deficits (i.e. smaller grey matter volumes) and functional overactivations in limbic regions that consistently include the hippocampus or the anterior cingulate cortex [17]. Maltreatment, even below the threshold of reportable childhood maltreatment, can lead to significant changes in the brain's emotion-regulating circuitry in adolescents [15]. In addition, chronic maltreatment is associated with cognitive deficits such as poorer inhibitory control [18] as described in adult first-degree relatives of AUD patients [19].

AUD adults indeed displayed grey matter volume (GMV) reductions in the hippocampus [20], amygdala [21], insula [22], caudate and putamen [23], cerebellum [24] as well as in the brainstem [25] compared to healthy controls using anatomical magnetic resonance imaging (MRI). GMV reductions were also observed in adolescents having a family history of AUD (called FH+) compared to those having no family history of AUD (called FH-) in the right amygdala [6], parahippocampal gyrus, thalamus, cingulum, superior frontal gyrus [7], orbitofrontal cortex [8] and cerebellum [9]. GMV reductions have also been reported in the parahippocampal gyrus [26] and in the amygdala [27] in $\mathrm{FH}+$ healthy adults. Interestingly, GMV reductions observed $\mathrm{FH}+$ individuals may indicate brain structural changes associated with a family history of AUD.

The most acknowledged mechanisms of vulnerability to addiction include functional deregulations of the reward system [28], intricately linked to deficits in inhibition [29] and sensationseeking [30]. Functional magnetic resonance imaging(fMRI) showed that $\mathrm{FH}+$ adolescents did not show variations of reward-related neural response [31,32] but had a blunted brain response to inhibition during Go/No-Go fMRI tasks [11,12,14]. However, shortterm detoxified AUD individuals [33] and $\mathrm{FH}+$ adults [34,35] had blunted activations in the ventral striatum during reward anticipation while performing a Monetary Incentive Delay (MID) task [36]. Elucidating whether reward processing in $\mathrm{FH}+$ individuals is affected at the earliest stages has been deemed essential to better characterize the addictive behaviour' predisposition.

Although some previous studies controlled (as exclusion criteria or confounding variable in the analyses) for the confounding effects of any psychiatric disorder and AUD [11,13], maternal AUD [8,13,32], foetal alcohol syndrome $[12,14,31]$ or even less frequently for maltreatment exposure [26], it is still unclear whether brain structural and functional features observed in $\mathrm{FH}+$ individuals persist when controlling simultaneously for these confounding factors. Moreover, there have been calls for neuroimaging research on brain structure in healthy FH+adults [19], since developmental maturation is still ongoing in adolescents [37]. In addition, previous studies on brain structure mostly predefined regions of interest, having possibly neglected some brain variations in $\mathrm{FH}+$ individuals. Finally, although deficits in differentiating a big versus small reward were reported in other addictive disorders [38] and impairments in the computation of expected reward value have been identified in AUD individuals [39,40], it is unknown whether they might contribute to vulnerability to AUD.

To address these limitations, the primary aim of the study was to explore the whole brain structure and processing of reward magnitude in adult first-degree relatives of AUD individuals having no prevalent confounding factors (i.e., severe maltreatment exposure, any clinically significant disorder, maternal AUD). The secondary aim was to assess inhibitory control to confirm inhibition deficits in a highly homogeneous sample. The Childhood Trauma Questionnaire (CTQ) score [41] was used as confounding variable in the statistical analyses. The tertiary aim was to explore on the whole brain the effects of low-to-moderate CTQ scores on brain structure and processing of reward in the $\mathrm{FH}+$ group.
Firstly, based on prior research, we hypothesized smaller GMV in $\mathrm{FH}+$ individuals in regions detailed above, i.e., amygdala, parahippocampal gyrus, thalamus, cingulum, superior frontal gyrus, orbitofrontal cortex and cerebellum, and deficits in neural response to a big versus small win during a MID task. Secondly, we hypothesized poorer inhibition in cognitive tests in $\mathrm{FH}+$ as compared to FH- participants. And thirdly, we hypothesized that exposure to low-to-moderate maltreatment can have a measurable impact on the brain i.e. structural deficits and reward-related overactivation in a group of $\mathrm{FH}+$ individuals.

\section{Material and methods}

\subsection{Participants' characteristics}

Participants were recruited in the Department of Psychiatry and Addictology of the Corentin-Celton Hospital (Issy-les-Moulineaux, France). Participants from the $\mathrm{FH}+$ group were mainly siblings of AUD patients followed in our department or participating in Alcoholics Anonymous, while participants from the FH- group were mainly psychology and medical students. The inclusion criteria were age ranging between 18 (age of civil majority) and 35, French native language, right-handed according to the Edinburgh Handedness Inventory scale. Healthy relatives of AUD individuals $(\mathrm{FH}+)$ were male and female defined as having two or more first or second-degree relatives with AUD including necessarily the father since inheritability to AUD has been found to be especially paternal [42] and excluding the mother to prevent foetal exposure to maternal AUD. Controls (FH-) were male and female defined as having no family history of AUD in neither first nor second-degree relatives.

Since we aimed to identify brain correlates of vulnerability to AUD rather than those of alcohol use per se, we considered the following exclusion criteria for the whole sample assessed using the Diagnostic Interview for Genetic Studies (DIGS $[43,44]$ ): past and current psychiatric diagnoses including abuse of or dependence to alcohol or other substances except tobacco according to DSM IV-TR criteria [45]. Current psychiatric diagnoses, including posttraumatic stress disorder, were also examined during a face-to-face interview with a psychiatrist. Using the DIGS, participants were also excluded if they fulfilled at least one of the following conditions: more than three alcohol intakes per week or more than six drinks per week for women or more than nine drinks per week for men, more than one episode of binge drinking (defined as drinking on the same occasion, i.e. at the same time or within a couple of hours of each other, five or more drinks for men and four or more drinks for women [46] over the past year and/or more than ten episodes in their lifetime; cannabis use in the last three months; use of prescribed and non-prescribed psychoactive drugs in the last six months; severe exposure to at least one form of childhood maltreatment, defined as having CTQ subscale scores $\geq 16, \geq 13, \geq 13, \geq 18$ and $\geq 13$ for emotional abuse, physical abuse, sexual abuse, emotional neglect, and physical neglect, respectively [47]. In addition to the excluded participants with maternal AUD, volunteers were not admitted to the study if they had a suspected foetal alcohol syndrome with generalized deficits in the processing and integration of information revealed by neurocognitive examination [48]. Exclusion criteria also involved other significant medical conditions such as medical events in perinatal history (perinatal foetal distress or prematurity of more than three weeks); any clinically significant or unstable disease; organic diseases affecting the central nervous system such as neurocognitive disorders, intellectual disabilities, history of head trauma with loss of consciousness and/or requiring hospitalization or meningoencephalitis; and MRI contra-indications. 
The protocol of the study was approved by the Ile-De-France VIII ethics committee and by the National Agency of Security for French biomedical researches. All participants signed an informed consent after receiving full information on the study. All procedures contributing to this work comply with the ethical standards of the relevant national and institutional committees on human experimentation and with the Helsinki Declaration of 1975, as revised in 2008.

\subsection{Data collection}

\subsubsection{Family history of AUD, clinical, sociodemographic and psychometric characteristics}

Assessments have been performed face-to-face by the investigators. Family history of AUD was examined using the Family Informant Schedule and Criteria (FISC). Axis I psychiatric and substance use disorders were assessed with the DIGS during a face-to-face interview with a psychiatrist. Age, sex and education (in number of years of schooling) were also collected. Maltreatment exposure was assessed with the CTQ. Sensation seeking was assessed with the Sensation-Seeking Scale-Form V of Zuckerman [49]. Trait impulsivity was assessed with the Barratt Impulsivity Scale ( $11^{\text {th }}$ version) [50].

\subsubsection{Magnetic resonance imaging (MRI)}

Acquisitions of high-resolution images were conducted with a 3.0 T Siemens Trio scanner and a 12-channel coil at CENIR-ICM platform, Pitié-Salpêtrière Hospital (Paris, France). Pre-processing steps and statistical analyses were run with the Statistical Parametric Mapping software (SPM12, Welcome Trust Centre for Neuroimaging, University College London, UK).

2.2.2.1. Structural brain imaging. The anatomical 3D-T1-weighted sequence was carried out with the following parameters: sagittal slice plane, Repetition Time $(\mathrm{TR})=2.3 \mathrm{~s}$, Echo Time $(\mathrm{TE})=2.93 \mathrm{~ms}$, $256 \times 256$ view matrix, 160 slices, voxel size $=1.1 \times 1.1 \times 1.1 \mathrm{~mm}$ and duration $=554 \mathrm{~s}$. A quality control has been conducted on T1 dataset from which images with a benign abnormality $(n=1)$, artifacts from excessive movements of the eyes's lens $(n=4)$ and head $(n=1)$ were excluded. The images were spatially normalized and segmented onto the MNI (Montreal Neurological Institute) tissue probability maps (TPM) into grey matter, white matter, cerebrospinal fluid, bone, soft tissue, background images - by fitting iteratively to TPM voxel's intensities [51]. Using the software $\mathrm{R}$, the package "mvoutlier" detected extreme values from a normal distribution (https://cran.rproject.org/web/packages/mvoutlier/index.html) leading to the exclusion of outlier volumes $(n=4)$. Then, covariances were calculated with the tool "check sample homogeneity" (http://www. neuro.uni-jena.de/vbm/check-sample-homogeneity/) and volumes with covariances greater than two standard deviations from the mean were detected with the R package "extreme values" (https:// cran.r-project.org/web/packages/extremevalues/index.html) thus outlier covariance volumes $(n=8)$ were excluded to control for errors of segmentation. Then, images were modulated and $10-\mathrm{mm}$ full-width at half-maximum (FWHM) Gaussian kernel smoothed. Overall, following visual quality control and outliers' detection, 18 (19.8\%) out of 91 acquisitions were excluded. The remaining sample for analysis was composed of $\mathrm{N}=73$ participants.

2.2.2.2. Functional brain imaging. An Echo-Planar Imaging sequence acquired 40 descending axial slices parallel to the anterior-posterior commissure for each $n=191$ time-series, with the following parameters: thickness $=2.4$ millimeters $(\mathrm{mm})$, $\mathrm{TR}=2.2 \mathrm{~s}, \quad \mathrm{TE}=30 \mathrm{~ms}$, matrix size $64 \times 64$, voxel size $=3.4 \times 3.4 \times 2.4 \mathrm{~mm}$ and duration $=427 \mathrm{~s}$. Excessive motion was prevented in the MRI with small wedges to fix head in place.
Participants' blood oxygen level-dependent (BOLD) signal time course was recorded while performing an event-related revised MID task in which the main difference to the original version [52] is the omission of loss trials [53]. This task displayed sequences of clue, target and feedback phases. The clue indicated the amount of the gain and the participants were instructed to respond upon appearance of the target which occurred $4 \mathrm{~s}$ after the clue. Response was followed by a $1.5 \mathrm{~s}$ visual feedback informing the participants of their trial result (Supplementary Fig. A.1). There were 42 trials (14 no wins; 14 small wins; 14 big wins).

A visual quality control has also been conducted on functional data. Images were excluded in cases of truncation $(n=1)$, signal abnormality $(n=2)$, major artefact $(n=1)$ and sequence error $(\mathrm{n}=1)$. No excessive motion was visually detected. Per participant, pre-processing steps included timing correction of the 40 slices per volume; rigid body realignment of the 191 volumes to the mean image; nonlinear normalization to MNI standardized space, intensity bias correction, writing of $3 \mathrm{~mm}^{3}$ voxel-wrapped images which were finally $10-\mathrm{mm}$ FWHM Gaussian filtered. Moreover, first-level analyses of BOLD signal changes were performed by adding anticipations and feedbacks as explanatory variables in the intra-subject model along with six motion realignment-computed parameters and derivatives. The canonical hemodynamic response function (HRF) accounted for lag between event onsets and effective BOLD signal changes. Participants not responding to all 'no win' conditions $(n=2)$ and with no or atypical BOLD signals in response to handgrip press were excluded $(n=6)$. Indeed, A quality control has been performed on fMRI "motor" contrasts (press left minus right, press right minus left) that usually displayed localized activations in the sensory / motor cortex and cerebellum. Therefore, any intense BOLD signal in the frontal, temporal or occipital lobe is atypical for motor contrasts (e.g. when the participant was not focusing on the task during the MID-task acquisition). Thus, out of the sample of 73 acquisitions, 13 (17.8\%) were removed. Overall, the fMRI sample available for the second-level analyses was composed of $\mathrm{N}=60$ participants.

\subsubsection{Cognitive assessments of inhibition}

Participants performed the following cognitive tests: Go/No-Go test [54] and Hayling test [55]. Further details are given in the Supplementary Appendix A.1.

\subsubsection{Statistical analyses}

For all statistical analyses, age, sex and CTQ total score were included as confounding variables to account for residual variance between and within groups.

2.2.4.1. Voxel-Based Morphometry analyses. Voxel-Based Morphometry (VBM) analysis of grey matter images consisted of between-group voxel-to-voxel $t$-tests across the whole brain. The global GMV was added in the statistical model [56]. We used the xjView toolbox (http://www.alivelearn.net/xjview) to localise the regions of difference. Statistical maps had a height threshold at $p<0.001$ uncorrected and an extent threshold at $p<0.05$ FamilyWise Error (FWE) corrected (cluster size $>520$ voxels). Because tobacco dependence [57] and cannabis use [58] may have confounding effects, we performed supplementary analyses with the number of pack-years of smoking or cannabis use as confounding variable.

We performed additional analyses to examine the confounding effects of the CTQ score on the GMV on the whole-brain within the $\mathrm{FH}+$ group. Significance was set at a height threshold at $p<0.05$ uncorrected and an extent threshold at $p<0.05$ FWE corrected (cluster size $>8000$ voxels). 
2.2.4.2. Functional brain imaging analyses. Between-group comparisons of BOLD signal maps were also performed as voxel-to-voxel $t$-tests across the whole brain for the contrast of anticipation of a "big versus small win" [53,59] to assess neural response to the processing of reward magnitude; as well as for the two conditions independently, i.e. anticipation of a big win and anticipation of a small win. Statistical maps had a height threshold at $p<0.001$ uncorrected and an extent threshold at $p<0.05$ FWE corrected (cluster size $>80$ voxels) [60]. The BrainVISA/Anatomist software (http://brainvisa.info) was used to build the figure which displays both structural and functional results. Because tobacco dependence [57] and cannabis use [58] might have confounding effects, we performed supplementary analyses with the number of pack-years of smoking or cannabis use as confounding variable.

The additional analyses to examine the confounding effects of the CTQ score on the BOLD signal within the FH+ group were significant at a height threshold at $p<0.05$ uncorrected and an extent threshold at $p<0.05$ FWE corrected (cluster size $>1250$ voxels).

2.2.4.3. Sociodemographic, psychometric and cognitive analyses. Between-group differences regarding individual factors were tested using unequal variance $t$-tests or chi-square tests when the dependent variable was continuous or categorical, respectively. Between-group multivariable analyses were performed using linear or logistic regression when the dependent variable was continuous or binary, respectively. Statistical significance for cognitive data was determined using a two-sided alpha a priori set at 0.0036 (0.05 Bonferroni corrected i.e. divided by the number of tests, i.e. 14).

\section{Results}

\subsection{Participants' characteristics}

There were 73 participants included in the psychometric, structural and cognitive analyses; 37 participants with a family history of alcohol use disorder (FH+) and 36 controls having no such history (FH-). Participants' characteristics are given in Table 1. The total duration of education showed a non-clinically significant mean difference of 1.0 year. We found no significant betweengroup differences in age, sex, and substance use (all $p>0.05$ ). However, we found a significantly greater severity of exposure to all childhood trauma subtypes in the $\mathrm{FH}+$ participants compared to the FH- controls, except for sexual abuse which was not significantly different between groups.

\subsection{Voxel-Based Morphometry analyses}

Between-group comparison of grey matter images showed that the $\mathrm{FH}+$ participants had significantly smaller GMV than the $\mathrm{FH}$ - controls in four clusters (Table 2; Fig. 1) comprising the right middle frontal gyrus, bilateral inferior frontal gyrus, right insula, bilateral nucleus accumbens, bilateral olfactory cortex, bilateral gyrus rectus, middle cingulate, bilateral precuneus and right pre- and post-central gyri. There was no region of larger GMV in the $\mathrm{FH}+$ participants compared to the FH- controls. These results were conserved when adding the number of pack-years of smoking or cannabis use as confounding variable (Supplementary Fig. B.1; Supplementary Fig. D.1).

The confounding effects of the CTQ score in the FH+ group are shown in Supplementary Fig. C.1; Supplementary Table A.1. There were significant negative associations between the CTQ score and GMV volumes in the bilateral hippocampus, left para-hippocampal gyrus and bilateral cerebellum.

\subsection{Functional brain imaging analyses}

There were 60 participants included in the fMRI analyses (31 $\mathrm{FH}+$ and $29 \mathrm{FH}-$ ). There was no significant between-group difference in their delayed final MID scores (means of $120.7 \mathrm{~s}$ $(\mathrm{SD}=35.1)$ and $114.3 \mathrm{~s}(\mathrm{SD}=28.1)$ for $\mathrm{FH}+$ and $\mathrm{FH}$ - participants, respectively; $t=-0.12 ; p=0.90)$. Between-group comparisons of neural response to a big versus small win indicated that the $\mathrm{FH}$ - controls had a significantly greater activation in the bilateral middle cingulum and right supplementary motor area (SMA) compared to the $\mathrm{FH}+$ participants (Table 3; Fig. 1). As to the brain

Table 1

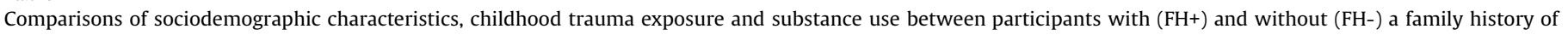
alcohol use disorder.

\begin{tabular}{|c|c|c|c|c|}
\hline \multirow[b]{2}{*}{ Sociodemographic characteristics } & \multirow{2}{*}{$\begin{array}{l}\text { FH+ } \text { participants }^{\text {a }}(n=37) \\
\text { Mean (SD) }\end{array}$} & \multirow{2}{*}{$\begin{array}{l}\text { FH- participants }{ }^{\text {a }}(n=36) \\
\text { Mean }(\text { SD })\end{array}$} & \multicolumn{2}{|c|}{$\mathrm{FH}+$ participants $v s \mathrm{FH}-$ participants } \\
\hline & & & $\mathbf{t}^{\mu}$ & $p$ value \\
\hline Age & $24.3(4.2)$ & $24.4(3.7)$ & -0.16 & 0.870 \\
\hline \multirow[t]{2}{*}{ Education (number of years of schooling) } & $13.9(2.1)$ & $14.9(2.0)$ & -2.22 & 0.030 \\
\hline & $\%$ & $\%$ & $x^{2 \beta}$ & $p$ value \\
\hline Sex (male) & 37.8 & 44.4 & 0.33 & 0.57 \\
\hline Childhood trauma exposure and substance use & Mean (SD) & Mean (SD) & $\mathbf{t}^{\mu}$ & $p$ value \\
\hline CTQ total score (mean (SD), minimum, maximum) & 46.6 (12.8), 27, 66 & 38.2 (7.0), 27, 53 & 3.46 & 0.001 \\
\hline Emotional abuse (mean (SD), minimum, maximum) & 9.0 (4.9), 5, 15 & $6.8(2.6), 5,14$ & 2.39 & 0.019 \\
\hline Emotional neglect (mean (SD), minimum, maximum) & $13.9(5.2), 5,17$ & 11.5 (3.9), 5,17 & 2.25 & 0.028 \\
\hline Physical abuse (mean (SD), minimum, maximum) & $6.4(2.7), 5,12$ & $5.2(0.6), 5,8$ & 2.57 & 0.013 \\
\hline Physical neglect (mean (SD), minimum, maximum) & $8.8(3.4), 5,12$ & $6.2(1.9), 5,12$ & 3.93 & $<\mathbf{0 . 0 0 1}$ \\
\hline Sexual abuse (mean (SD), minimum, maximum) & $8.5(1.2), 6,10$ & $8.6(1.1), 6,10$ & -0.16 & 0.875 \\
\hline Number of standard alcoholic drinks per week & $2.2(3.0)$ & $1.8(1.8)$ & 0.57 & 0.570 \\
\hline \multirow[t]{2}{*}{ Number of pack-years of smoking } & $2.3(3.4)$ & $1.7(3.0)$ & 0.83 & 0.410 \\
\hline & $\%$ & $\%$ & $x^{2 \beta}$ & $p$ value \\
\hline Smokers & 37.8 & 50.0 & 1.10 & 0.295 \\
\hline Lifetime cannabis use & 73.0 & 66.7 & 0.35 & 0.557 \\
\hline Lifetime use of any other drugs & 27.0 & 25.0 & 0.04 & 0.844 \\
\hline
\end{tabular}

a Participants with a family history of alcohol use disorder had to have two or more first or second-degree relatives with alcohol use disorder (FH+) - including necessarily the father and excluding the mother. Controls had to have no family history of alcohol use disorder in neither first nor second degree relatives (FH-).

$\mu$ Unequal variance t-tests $(\mathrm{df}=71)$.

${ }^{\beta} \mathrm{x}^{2}$ tests $(\mathrm{df}=1)$. Continuous variables are presented as their mean values and standard deviation (SD). Categorical variables are presented as percentages. $p$ values in bold are statistically significant $(p<0.05)$. 
Table 2

Regions of reduction in grey matter volume in the participants having a family history of alcohol use disorder (FH+) compared to controls (FH-).

\begin{tabular}{|c|c|c|c|c|c|c|c|c|}
\hline \multirow[t]{2}{*}{ Region } & \multirow[b]{2}{*}{ BA } & \multicolumn{3}{|l|}{ Cluster level } & \multirow{2}{*}{$\begin{array}{l}\text { Peak level } \\
p \text { value } \\
\text { uncorrected }\end{array}$} & \multicolumn{2}{|c|}{ MNI Coordinates } & \multirow[b]{2}{*}{$\mathrm{z}$} \\
\hline & & $\begin{array}{l}\text { Cluster size } \\
\text { (in voxels) }\end{array}$ & $\begin{array}{l}p \text { value } \\
\text { FWE corrected }\end{array}$ & $T$ & & $\mathrm{x}$ & $\mathrm{y}$ & \\
\hline \multicolumn{9}{|l|}{ Cluster 1} \\
\hline $\mathrm{R}$ middle frontal gyrus & 9 & 1368 & $4.4072 \mathrm{e}-04$ & 5.97 & $5.2166 \mathrm{e}-08$ & 28 & 40 & 34 \\
\hline \multicolumn{9}{|l|}{$\mathrm{R}$ inferior frontal gyrus } \\
\hline Orbital part & 11 & & & 4.80 & $4.7218 \mathrm{e}-06$ & 44 & 48 & -9 \\
\hline Triangular part & 45 & & & 3.89 & $1.1968 \mathrm{e}-04$ & 46 & 44 & 0 \\
\hline \multicolumn{9}{|l|}{ Cluster 2} \\
\hline \multicolumn{9}{|l|}{$\mathrm{R}$ inferior frontal gyrus } \\
\hline Opercular part & 47 & 4599 & $1.5614 \mathrm{e}-09$ & 5.79 & $1.0702 e-07$ & 48 & 10 & 2 \\
\hline Triangular part & & & & 4.36 & $2.2891 \mathrm{e}-05$ & 56 & 22 & 14 \\
\hline L inferior frontal gyrus, Orbital part & 11 & & & 4.23 & $3.6739 \mathrm{e}-05$ & -16 & 10 & -20 \\
\hline $\mathrm{R}$ olfactory cortex & 47 & & & 5.04 & $1.9367 e-06$ & 18 & 10 & -18 \\
\hline L olfactory cortex & 32 & & & 4.59 & $1.0342 \mathrm{e}-05$ & -6 & 20 & -10 \\
\hline $\mathrm{R}$ insula & & & & 4.82 & $4.3244 \mathrm{e}-06$ & 40 & -4 & 8 \\
\hline L gyrus rectus & 11 & & & 4.18 & $4.4344 \mathrm{e}-05$ & -6 & 33 & -16 \\
\hline $\mathrm{R}$ gyrus rectus & 11 & & & 4.15 & $4.8556 \mathrm{e}-05$ & 4 & 44 & -21 \\
\hline $\mathrm{R}$ nucleus accumbens & & & & 4.51 & $1.3629 \mathrm{e}-05$ & 8 & 10 & -10 \\
\hline L nucleus accumbens & & & & 4.17 & $4.4885 \mathrm{e}-05$ & -6 & 12 & -8 \\
\hline \multicolumn{9}{|l|}{ Cluster 3} \\
\hline Cingulum middle/posterior & 31 & 1582 & $1.5793 e-04$ & 4.92 & $2.9949 \mathrm{e}-06$ & -2 & -44 & 33 \\
\hline $\mathrm{R}$ precuneus & 7 & & & 4.62 & $9.2786 \mathrm{e}-06$ & 4 & -54 & 21 \\
\hline L precuneus & 31 & & & 4.21 & $3.8984 \mathrm{e}-05$ & -4 & -57 & 32 \\
\hline R Calcarine fissure/lingual & & & & 3.69 & $2.2512 \mathrm{e}-04$ & 10 & -60 & 10 \\
\hline \multicolumn{9}{|l|}{ Cluster 4} \\
\hline R precentral gyus & 4 & 676 & 0.0192 & 4.47 & $1.5681 \mathrm{e}-05$ & 44 & -15 & 44 \\
\hline $\mathrm{R}$ middle frontal gyrus & 6 & & & 4.17 & $4.5851 \mathrm{e}-05$ & 39 & -3 & 54 \\
\hline R postcentral gyrus & & & & 3.36 & $6.4635 e-04$ & 54 & -8 & 36 \\
\hline
\end{tabular}

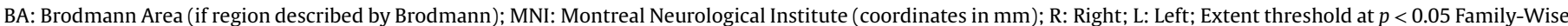

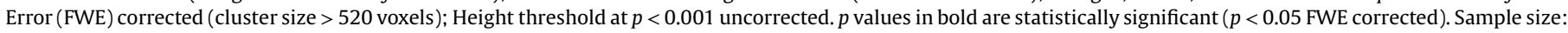
$\mathrm{n}=37 \mathrm{FH}+$ and $\mathrm{n}=36 \mathrm{FH}-$.

activity in response to anticipation of a small win, the $\mathrm{FH}+$ participants exhibited a significant greater activity than the FH- controls in the anterior cingulum (Table 3; Fig. 1). As to the brain activity in response to anticipation of a big win, no significant difference was observed between the groups. The same results were found when adding the number of pack-years of smoking or cannabis use as confounding variable (Supplementary Fig. B.2; Supplementary Fig. D.2).

The confounding effects of the CTQ total score in the FH+ group are shown in Supplementary Fig. C.2; Supplementary Table A.2. There were significant positive associations between the CTQ score and brain activation during the processing of reward magnitude in the bilateral parahippocampal gyrus, bilateral fusiform and lingual gyri, left cerebellum and right inferior frontal gyrus.

\subsection{Cognitive measures}

No significant between-group differences in the sensationseeking and trait impulsivity scores were observed $(p<0.001)$ (Table 4). In the Hayling test, compared to the FH- controls, the $\mathrm{FH}+$ participants showed significant increases in time response per sentence and corrected time response, and a significant lower number of accurate responses. In the Go/No-Go test, the $\mathrm{FH}+$ participants made significantly more errors of commission and omission than the FH- controls $(p<0.001)$ (Table 4$)$.

\section{Discussion}

This study sought to determine whether healthy adult firstdegree relatives of individuals with AUD $(\mathrm{FH}+)$ differ from controls having no family history of AUD (FH-) in grey matter volumes (GMV), in brain response to a big versus small win during a Monetary
Incentive Delay (MID) task, and in inhibition performance (Hayling and Go/No-Go tests). We ruled out the confounding effects of childhood maltreatment, and of any psychiatric disorder or AUD, and of prenatal exposure to maternal AUD. We found that family history of AUD was associated with i) smaller GMV in the frontal and cingulate regions as well as in the bilateral nucleus accumbens and right insula, ii) a blunted activation in the middle cingulum during the processing of reward magnitude and a greater activation in the anterior cingulum in response to anticipation of a small win, iii) and deficits in the accuracy of the inhibition processes and in the latency of the act of control. Overall, the present findings confirm and extend research on vulnerability to AUD.

On one hand, our findings are in line with previous studies on $\mathrm{FH}+$ adolescents that showed localised GMV deficits compared to controls [6-9]. As previously identified in two distinct studies in $\mathrm{FH}+$ adolescents $[7,14]$, in the present study the middle cingulum was explicitly at the intersect of the brain structural (i.e. smaller GMV) and functional (i.e. blunted activation) differences between the $\mathrm{FH}+$ and $\mathrm{FH}-$ participants. In healthy individuals, the volume of the middle cingulum underlies conflict monitoring [61] while its activity is typically involved during cognitive processing tasks [62].

On the other hand, we did not find smaller hippocampus in the $\mathrm{FH}+$ participants when controlling for low-to-moderate exposure to childhood maltreatment as confounding variable (i.e variable of no interest in the statistical analyses), unlike previous studies conducted in $\mathrm{FH}+$ adolescents [7], FH+adults [26] and AUD individuals [20]. It remains unknown whether previous findings of hippocampal grey matter volume reductions were due to childhood trauma. However, childhood maltreatment exposure is known to impact the developing brain in numerous brain regions, the most consistently reported being the hippocampus as well as the anterior cingulate cortex [17]; and our additional findings 

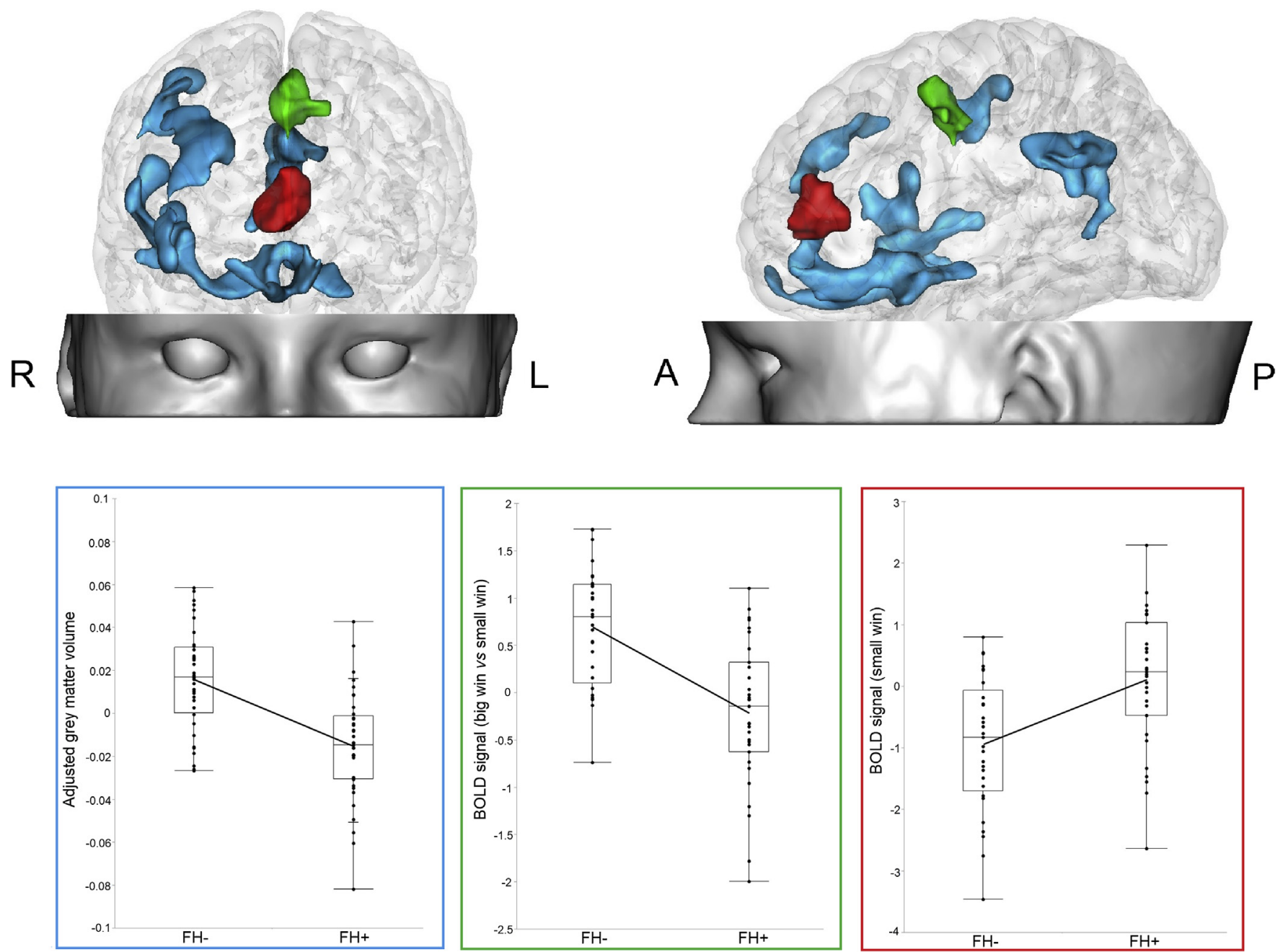

Fig. 1. Brain regions of differences in grey matter volumes and bold signal changes during anticipation of reward in a MID task between individuals with and without a family history of alcohol use disorder.

Participants with a positive family history of alcohol use disorder $(\mathrm{FH}+)$ presented smaller grey matter volumes in four clusters (blue colour) compared to participants having no family history of alcohol use disorder ( $\mathrm{FH}-$ ). $\mathrm{FH}+$ individuals also had a blunted activation in response to a big versus small win (green colour) and a greater activation during anticipation of a small win (red colour) compared to FH- controls during the MID anticipation phase. Extent threshold was set at $p<0.05$ Family-Wise Error (FWE) corrected; Height threshold was set at $p<0.001$ uncorrected. Each point on the boxplots represents the adjusted (for age, sex and Childhood Trauma Questionnaire total score) mean value for each participant. The line joining the boxplots spots the group mean. Orientation R: Right; L: Left; A: Anterior; P: Posterior; voxel resolution $1.5 \times 1.5 \times 1.5 \mathrm{~mm}$; Montreal Neurological Institute space. Sample size: $n=37 \mathrm{FH}+$ and $\mathrm{n}=36 \mathrm{FH}-$ in the structural analyses; $\mathrm{n}=31 \mathrm{FH}+$ and $\mathrm{n}=29 \mathrm{FH}-$ individuals in the functional analyses.

Table 3

Regions of BOLD signal changes in participants having a family history of alcohol use disorder (FH+) compared to controls (FH-) during a Monetary Incentive Delay (MID) task.

\begin{tabular}{|c|c|c|c|c|c|c|c|c|}
\hline \multirow[t]{2}{*}{ Region } & \multirow[t]{2}{*}{ BA } & \multirow{2}{*}{$\begin{array}{l}\text { Cluster level } \\
\text { Cluster Size } \\
\text { (in voxels) }\end{array}$} & \multirow[b]{2}{*}{$\begin{array}{l}\mathrm{p} \text { value } \\
\text { FWE corrected }\end{array}$} & \multirow[t]{2}{*}{$T$} & \multirow{2}{*}{$\begin{array}{l}\text { Peak Level } \\
\text { p value } \\
\text { uncorrected }\end{array}$} & \multicolumn{3}{|c|}{ MNI Coordinates } \\
\hline & & & & & & $\mathrm{x}$ & $\mathrm{y}$ & $\mathrm{z}$ \\
\hline \multicolumn{9}{|c|}{$\begin{array}{l}\text { Activation in } \mathrm{FH} \text { - greater than in } \mathrm{FH}^{+} \\
\text {Big win versus small win }\end{array}$} \\
\hline $\mathrm{R}$ supplementary motor area & 6 & 89 & 0.037 & 4.55 & $1.5088 \mathrm{e}-05$ & 6 & -1 & 53 \\
\hline $\mathrm{R}$ middle cingulum & & & & 4.53 & $1.5867 \mathrm{e}-05$ & 6 & -7 & 47 \\
\hline L middle cingulum & & & & 3.56 & $3.8850 \mathrm{e}-04$ & -6 & -4 & 44 \\
\hline \multicolumn{9}{|c|}{$\begin{array}{l}\text { Activation in } \mathrm{FH}+\text { greater than in } \mathrm{FH}- \\
\text { Anticipation of a small win }\end{array}$} \\
\hline Anterior cingulum & & 63 & 0.157 & 4.80 & $6.3054 \mathrm{e}-06$ & 0 & 29 & 2 \\
\hline
\end{tabular}

BA: Brodmann Area (if region described by Brodmann); MNI: Montreal Neurological Institute (coordinates in mm); R: Right; L: Left; Extent threshold at $p<0.05$ Family-Wise Error (FWE) corrected (cluster size $>80$ voxels); Height threshold at $p<0.001$ uncorrected. $p$ values in bold are statistically significant $(p<0.05$ FWE corrected). Sample size: $\mathrm{n}=31 \mathrm{FH}+$ and $\mathrm{n}=29 \mathrm{FH}-$. 
Table 4

Comparisons of personality and cognitive measures between participants with (FH+) and without (FH-) a family history of alcohol use disorder.

\begin{tabular}{|c|c|c|c|c|}
\hline & \multirow{2}{*}{$\begin{array}{l}\text { FH+ participants a }(n=37) \\
\text { Mean }(S D)\end{array}$} & \multirow{2}{*}{$\begin{array}{l}\text { FH- participants }{ }^{\text {a }}(n=36) \\
\text { Mean }(S D)\end{array}$} & \multicolumn{2}{|c|}{$\mathrm{FH}+$ participantsvs $\mathrm{FH}-$ participants } \\
\hline & & & $\beta(\mathrm{SE})^{\mu}$ & $p$ value ${ }^{\mu}$ \\
\hline \multicolumn{5}{|l|}{ Sensation Seeking Scale } \\
\hline Total score & $20.9(5.7)$ & $20.9(6.7)$ & $-0.83(1.57)$ & 0.599 \\
\hline Thrill seeking & $6.2(2.5)$ & $6.7(2.9)$ & $-0.85(0.68)$ & 0.214 \\
\hline Inhibition & $4.4(2.4)$ & $4.6(2.6)$ & $-0.36(0.63)$ & 0.568 \\
\hline Experience & $6.6(1.9)$ & $6.3(1.9)$ & $0.21(0.49)$ & 0.678 \\
\hline Boredom Susceptibility & $3.8(2.1)$ & $3.3(2.0)$ & $0.18(0.52)$ & 0.727 \\
\hline \multicolumn{5}{|l|}{ Barratt Impulsiveness Scale } \\
\hline Total score & $63.6(10.4)$ & $61.1(7.0)$ & $1.60(2.22)$ & 0.475 \\
\hline Attentional score & $17.1(3.4)$ & $16.5(3.0)$ & $0.04(0.81)$ & 0.960 \\
\hline Motor score & $22.2(4.3)$ & $17.1(3.4)$ & $0.52(1.05)$ & 0.620 \\
\hline Nonplanning score & $24.3(4.8)$ & $23.3(2.6)$ & $1.03(0.97)$ & 0.292 \\
\hline \multicolumn{5}{|l|}{ Hayling Sentence Completion Test } \\
\hline Time response per sentence (seconds) & $7.9(2.1)$ & $5.8(0.9)$ & $1.92(0.41)$ & $<\mathbf{0 . 0 0 1}$ \\
\hline Corrected time response & $88.9(52.6)$ & $32.1(11.6)$ & 55.49 (9.97) & $<\mathbf{0 . 0 0 1}$ \\
\hline Penalties & $9.2(7.4)$ & $5.8(5.4)$ & $4.60(1.66)$ & 0.007 \\
\hline Number of accurate responses & $8.0(3.9)$ & $13.4(6.3)$ & $-5.92(1.34)$ & $<\mathbf{0 . 0 0 1}$ \\
\hline \multicolumn{5}{|l|}{ Go/No-Go Test } \\
\hline Total number of errors of commission and omission & $1.6(1.4)$ & $0.5(0.7)$ & $1.17(0.30)$ & $<\mathbf{0 . 0 0 1}$ \\
\hline
\end{tabular}

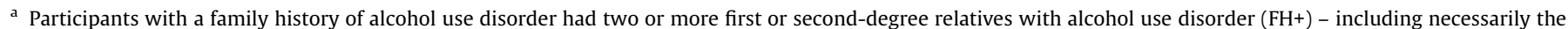
father and excluding the mother. Controls had no family history of alcohol use disorder in neither first nor second degree relatives (FH-). SD: standard deviation.

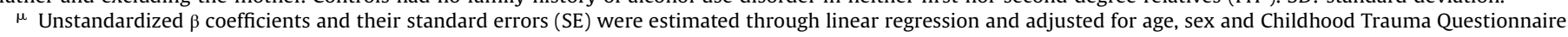
total score $(\mathrm{df}=4)$. $p$ values in bold are statistically significant (alpha set $a$ priori at $0.05 / 14=0.0036$ Bonferroni corrected).

suggest that low-to-moderate maltreatment exposure may have an impact in $\mathrm{FH}+$ participants on both brain structure and activity in the hippocampal and cerebellar regions.

Using the whole-brain method instead of the region-of-interest approach, additional brain regions were detected compared to earlier studies with $\mathrm{FH}+$ individuals. We found that the $\mathrm{FH}$ + participants had smaller GMV in the bilateral nucleus accumbens, a core region of the reward system involved in the addictive processes. Prior research conducted among AUD individuals showed GMV reductions in the caudate nucleus and putamen compared to healthy controls [23], supporting the hypothesis that this variation might pre-exist in the vulnerable individuals. Similarly, the insula, part of the paralimbic region, was altered in the present sample of $\mathrm{FH}+$ participants on the right side, as previously described in AUD individuals [22] and tobacco smokers [63,64].

Despite similar MID-task performance between groups regarding brain function, the $\mathrm{FH}+$ participants had a blunted activation in the middle cingulum in response to the processing of reward magnitude. Deficits in the computation of expected values have been linked to impaired reward-based decision making [65] thus may contribute to vulnerability to AUD. However, the $\mathrm{FH}+$ individuals showed an overactivity of the anterior cingulum compared to the FH-controls; a region that is thought to translate neural signals related to degree of reward expectancy [66] and is involved in the reward system [28]. Among detoxified AUD individuals, self-efficacy to abstain was positively associated with the neural response in the anterior cingulum during the MID anticipation phase [67], thus this overaction might denote a protective factor to AUD.

Deregulations of the inhibitory control have been largely reported in functional studies in $\mathrm{FH}+$ adolescents $[11,12,14]$. We confirmed that the $\mathrm{FH}+$ group had deficits compared to the FHgroup in both cognitive tasks of inhibition, supporting the validity of the present sample. Deficient inhibitory control indicates poorer resistance to distractor or proactive interference [68], leading to a greater likelihood that a response will be executed rather than withheld [69]. Although inhibitory control has been identified as a main component of impulsivity [70], we did not find significant between-group differences neither for the Barratt Impulsivity
Scale score nor for the Sensation-Seeking Scale score that are associated with substance misuse in young individuals [53]. Hence, the absence of such personality variations might denote a protective factor to AUD in the healthy $\mathrm{FH}+$ individuals.

Despite the strengths of the study design involving participants with fewer confounding factors than previous studies, this design aiming to examine more specifically brain correlates of vulnerability to AUD, had some limitations. Firstly, there was no group of AUD individuals to compare with the $\mathrm{FH}+$ participants. This would allow to disentangle the neural correlates of vulnerability to AUD from the mixed effects of alcohol intoxication observed in AUD individuals [5]. Secondly, although we excluded participants with severe exposure to any childhood maltreatment type, $\mathrm{FH}+$ participants still had substantially higher CTQ scores than FH- participants. Thirdly, we excluded participants with AUD and maternal AUD exposure, however we cannot exclude potential brain and/or cognitive alterations due to past minimal alcohol use or minimal foetal alcohol exposure. Fourthly, psychiatric disorders were assessed following DSM-IV-TR criteria and not DSM-5 criteria. Fifthly, future studies with greater statistical power would benefit in examining whether our results hold or differ in women and men. Finally, we cannot exclude that observed differences may reflect protective factors in the FH- group instead of vulnerability factors in the $\mathrm{FH}+$ group.

\section{Conclusions}

We showed that adult first-degree relatives of AUD individuals display GMV reductions in the frontal and cingulate regions, as well as a blunted activation in the middle cingulum in response to the processing of reward magnitude during a MID task. Moreover, our findings highlight pre-existing structural deficits in the nucleus accumbens and right insula, and an overactivation in the anterior cingulum in response to anticipation of small non-drug rewards. This study confirms and extents prior research on relatives of AUD individuals, and encourages to systematically control for the confounding effect of childhood maltreatment among other confounders, i.e. any clinically significant disorder and exposure to maternal AUD, in the study of vulnerability to AUD. 


\section{Declaration of Competing Interest}

Frédéric Limosin has received speaker and consulting fees from Astra Zeneca, Euthérapie-Servier, Janssen, Lundbeck, Otsuka Pharmaceuticals and Roche. The present work is unrelated to these relationships. Other authors have no conflicts of interest or financial disclosures to make.

\section{Acknowledgements}

This research was supported by the French Ministery of Health (Programme Hospitalier de Recherche Clinique P110132, 2011), the Fondation pour la Recherche Médicale (DPA20140629802, 2014) and the Fédération pour la Recherche sur le Cerveau (C16/0932A01, 2016). The study was sponsored by Assistance Publique Hopitaux de Paris, represented by the HEGP Clinical Research Unit (Pr Gilles Chatellier). We would like to thank the team of CENIR - Centre for NeuroImaging Research, ICM - Institut du Cerveau et de la Moelle épinière, Hôpital Pitié-Salpêtrière, Paris, France. We would also like to thank the IMAGEN consortium that provided the revised version of the Monetary Incentive Delay task and Joelle Francis for her advices.

\section{Appendix A. Supplementary data}

Supplementary material related to this article can be found, in the online version, at doi:https://doi.org/10.1016/j.eurpsy.2019.08.003.

\section{References}

[1] Gowing LR, Ali RL, Allsop S, Marsden J, Turf EE, West R, et al. Global statistics on addictive behaviours: 2014 status report. Addiction 2015;110(June (6)):904-19.

[2] Bienvenu OJ, Davydow DS, Kendler KS. Psychiatric ‘diseases’ versus behavioral disorders and degree of genetic influence. Psychol Med 2011;41(January (1)):33-40.

[3] Osby U, Liljenberg J, Kockum I, Gunnar A, Terenius L. Genes and alcohol. Eur Psychiatry 2010;25(June (5)):281-3.

[4] Nurnberger JI, Wiegand R, Bucholz K, O'Connor S, Meyer ET, Reich T, et al. A family study of alcohol dependence: coaggregation of multiple disorders in relatives of alcohol-dependent probands. Arch Gen Psychiatry 2004;61 (December (12)):1246-56.

[5] Dupuy M, Chanraud S. Imaging the addicted brain: alcohol. Int Rev Neurobiol 2016;129:1-31.

[6] Hill SY, De Bellis MD, Keshavan MS, Lowers L, Shen S, Hall J, et al. Right amygdala volume in adolescent and young adult offspring from families at high risk for developing alcoholism. Biol Psychiatry 2001;49(June (11)):894-905.

[7] Benegal V, Antony G, Venkatasubramanian G, Jayakumar PN. Gray matter volume abnormalities and externalizing symptoms in subjects at high risk for alcohol dependence. Addict Biol 2007;12(March (1)):122-32.

[8] Hill SY, Wang S, Kostelnik B, Carter H, Holmes B, McDermott M, et al. Disruption of orbitofrontal cortex laterality in offspring from multiplex alcohol dependence families. Biol Psychiatry 2009;65(January (2)):129-36.

[9] Hill SY, Wang S, Carter H, Tessner K, Holmes B, McDermott M, et al. Cerebellum volume in high-risk offspring from multiplex alcohol dependence families: association with allelic variation in GABRA2 and BDNF. Psychiatry Res 2011;194(December (3)):304-13.

[10] Cservenka A. Neurobiological phenotypes associated with a family history of alcoholism. Drug Alcohol Depend 2016;1(January (158)):8-21.

[11] Schweinsburg AD, Paulus MP, Barlett VC, Killeen LA, Caldwell LC, Pulido C, et al. An FMRI study of response inhibition in youths with a family history of alcoholism. Ann N Y Acad Sci 2004;1021(June):391-4.

[12] Heitzeg MM, Nigg JT, W-YW Yau, Zucker RA, Zubieta J-K. Striatal dysfunction marks preexisting risk and medial prefrontal dysfunction is related to problem drinking in children of alcoholics. Biol Psychiatry 2010;68 (August (3)):287-95.

[13] Cservenka A, Nagel BJ. Risky decision-making: an FMRI study of youth at high risk for alcoholism. Alcohol Clin Exp Res 2012;36(April (4)):604-15.

[14] Hardee JE, Weiland BJ, Nichols TE, Welsh RC, Soules ME, Steinberg DB, et al. Development of impulse control circuitry in children of alcoholics. Biol Psychiatry 2014;76(November (9)):708-16.

[15] Herringa RJ, Birn RM, Ruttle PL, Burghy CA, Stodola DE, Davidson RJ, et al. Childhood maltreatment is associated with altered fear circuitry and increased internalizing symptoms by late adolescence. Proc Natl Acad Sci USA 2013;110 (November (47)):19119-24.

[16] Keyes KM, Eaton NR, Krueger RF, McLaughlin KA, Wall MM, Grant BF, et al. Childhood maltreatment and the structure of common psychiatric disorders. Br J Psychiatry 2012;200(February (2)):107-15.
[17] Teicher MH, Samson JA, Anderson CM, Ohashi K. The effects of childhood maltreatment on brain structure, function and connectivity. Nat Rev Neurosci 2016;17(10):652-66.

[18] Cowell RA, Cicchetti D, Rogosch FA, Toth SL. Childhood maltreatment and its effect on neurocognitive functioning: timing and chronicity matter. Dev Psychopathol 2015;27(May (2)):521-33.

[19] Gierski F, Hubsch B, Stefaniak N, Benzerouk F, Cuervo-Lombard C, Bera-Potelle C, et al. Executive functions in adult offspring of alcohol-dependent probands: toward a cognitive endophenotype? Alcohol Clin Exp Res 2013;37(January Suppl. 1):E356-363.

[20] Beresford TP, Arciniegas DB, Alfers J, Clapp L, Martin B, Du Y, et al Hippocampus volume loss due to chronic heavy drinking. Alcohol Clin Exp Res 2006;30(November (11)):1866-70.

[21] Wrase J, Makris N, Braus DF, Mann K, Smolka MN, Kennedy DN, et al. Amygdala volume associated with alcohol abuse relapse and craving. Am J Psychiatry 2008;165(September (9)):1179-84.

[22] Chanraud S, Martelli C, Delain F, Kostogianni N, Douaud G, Aubin H-J, et al Brain morphometry and cognitive performance in detoxified alcoholdependents with preserved psychosocial functioning. Neuropsychopharmacology 2007;32(February (2)):429-38.

[23] Sullivan EV, Deshmukh A, De Rosa E, Rosenbloom MJ, Pfefferbaum A. Striatal and forebrain nuclei volumes: contribution to motor function and working memory deficits in alcoholism. Biol Psychiatry 2005;57(April (7)):768-76.

[24] Sullivan EV, Deshmukh A, Desmond JE, Mathalon DH, Rosenbloom MJ, Lim KO, et al. Contribution of alcohol abuse to cerebellar volume deficits in men with schizophrenia. Arch Gen Psychiatry 2000;57(September (9)):894-902.

[25] Pfefferbaum A, Rosenbloom M, Serventi KL, Sullivan EV. Corpus callosum, pons, and cortical white matter in alcoholic women. Alcohol Clin Exp Res 2002;26(March (3)):400-6.

[26] Sjoerds Z, M-JV Tol, van den Brink W, der Wee NJAV, Buchem MAV, Aleman A, et al. Family history of alcohol dependence and gray matter abnormalities in non-alcoholic adults. World J Biol Psychiatry 2013;14(December (8)):565-73.

[27] Dager AD, McKay DR, Kent JW, Curran JE, Knowles E, Sprooten E, et al. Shared genetic factors influence amygdala volumes and risk for alcoholism. Neuropsychopharmacology 2015;40(January (2)):412-20.

[28] Volkow ND, Morales M. The brain on drugs: from reward to addiction. Cell 2015;162(August (4)):712-25

[29] Churchwell JC, Yurgelun-Todd DA. Neuroimaging, adolescence, and risky behavior. Inhibitory control and drug abuse prevention. New York, NY: Springer; 2011. p. 101-22.

[30] Cservenka A, Herting MM, Seghete KLM, Hudson KA, Nagel BJ. High and low sensation seeking adolescents show distinct patterns of brain activity during reward processing. Neuroimage 2013;1(February (66)):184-93.

[31] Bjork JM, Knutson B, Hommer DW. Incentive-elicited striatal activation in adolescent children of alcoholics. Addiction 2008;103(August (8)):1308-19.

[32] Müller KU, Gan G, Banaschewski T, Barker GJ, Bokde ALW, Büchel C, et al. No differences in ventral striatum responsivity between adolescents with a positive family history of alcoholism and controls. Addict Biol 2015;20(May (3)):534-45.

[33] Beck A, Schlagenhauf F, Wüstenberg T, Hein J, Kienast T, Kahnt T, et al. Ventral striatal activation during reward anticipation correlates with impulsivity in alcoholics. Biol Psychiatry 2009;66(October (8)):734-42.

[34] Andrews MM, Meda SA, Thomas AD, Potenza MN, Krystal JH, Worhunsky P, et al. Individuals family history positive for alcoholism show functional magnetic resonance imaging differences in reward sensitivity that are related to impulsivity factors. Biol Psychiatry 2011;69(April (7)):675-83.

[35] W-YW Yau, Zubieta J-K, Weiland BJ, Samudra PG, Zucker RA, Heitzeg MM. Nucleus accumbens response to incentive stimuli anticipation in children of alcoholics: relationships with precursive behavioral risk and lifetime alcohol use. J Neurosci 2012;32(February (7)):2544-51.

[36] Balodis IM, Potenza MN. Anticipatory reward processing in addicted populations: a focus on the monetary incentive delay task. Biol Psychiatry 2015;77(March (5)):434-44.

[37] Mills KL, Tamnes CK. Methods and considerations for longitudinal structural brain imaging analysis across development. Dev Cogn Neurosci 2014;9 (July):172-90.

[38] Lole L, Gonsalvez CJ. Does size matter? An examination of problem gamblers' skin conductance responses to large and small magnitude rewards. Psychophysiology 2017;54(October (10)):1541-8.

[39] Hariri AR, Brown SM, Williamson DE, Flory JD, de Wit H, Manuck SB. Preference for immediate over delayed rewards is associated with magnitude of ventral striatal activity. J Neurosci 2006;26(December (51)):13213-7.

[40] Grodin EN, Steckler LE, Momenan R. Altered striatal response during effortbased valuation and motivation in alcohol-dependent individuals. Alcohol Alcohol 2016;51(November (6)):638-46.

[41] Bernstein DP, Stein JA, Newcomb MD, Walker E, Pogge D, Ahluvalia T, et al. Development and validation of a brief screening version of the Childhood Trauma Questionnaire. Child Abuse Negl 2003;27(February (2)):169-90.

[42] McGue M, Iacono WG, Legrand LN, Elkins I. Origins and consequences of age at first drink. II. Familial risk and heritability. Alcohol Clin Exp Res 2001;25 (August (8)):1166-73.

[43] Nurnberger JI, Blehar MC, Kaufmann CA, York-Cooler C, Simpson SG, HarkavyFriedman J, et al. Diagnostic interview for genetic studies. Rationale, unique features, and training. NIMH Genetics Initiative. Arch Gen Psychiatry 1994;51 (November (11)849-59 discussion 863-864. 
[44] Preisig M, Fenton BT, Matthey ML, Berney A, Ferrero F. Diagnostic interview for genetic studies (DIGS): inter-rater and test-retest reliability of the French version. Eur Arch Psychiatry Clin Neurosci 1999;249(4):174-9.

[45] American Psychiatric Association. Diagnostic and statistical manual of mental disorders: DSM-IV. 4th ed revised Washington, DC: American Psychiatric Publishing; 2000.

[46] National Institute on Alcohol Abuse and Alcoholism. Winter). NIAAA council approves definition of binge drinking. NIAAA newsletter 2004; Vol. 3:. p. 3. . Retrieved from https://pubs.niaaa.nih.gov/publications/Newsletter/ winter2004/Newsletter_Number3.pdf.

[47] Bernstein D, Fink L. Manual for the childhood trauma questionnaire. New York: The Psychological Corporation; 1998.

[48] Kodituwakku PW. Neurocognitive profile in children with fetal alcohol spectrum disorders. Dev Disabil Res Rev 2009;15(3):218-24.

[49] Loas G, Verrier A, Flament MF, Perez-Diaz F, Corcos M, Halfon O, et al. Factorial structure of the Sensation-Seeking Scale-Form V: confirmatory factorial analyses in nonclinical and clinical samples. Can J Psychiatry 2001;46 (November (9)):850-5.

[50] Baylé FJ, Bourdel MC, Caci H, Gorwood P, Chignon JM, Adés J, et al. Factor analysis of french translation of the Barratt impulsivity scale (BIS-10). Can J Psychiatry 2000;45(March (2)):156-65.

[51] Ashburner J, Friston KJ. Voxel-based morphometry-the methods. Neuroimage 2000;11(June (6 Pt 1)):805-21.

[52] Knutson B, Westdorp A, Kaiser E, Hommer D. FMRI visualization of brain activity during a monetary incentive delay task. Neuroimage 2000;12(July (1)):20-7.

[53] Nees F, Tzschoppe J, Patrick CJ, Vollstädt-Klein S, Steiner S, Poustka L, et al. Determinants of early alcohol use in healthy adolescents: the differential contribution of neuroimaging and psychological factors. Neuropsychopharmacology 2012;37(March (4)):986-95.

[54] Kamarajan C, Porjesz B, Jones KA, Choi K, Chorlian DB, Padmanabhapillai A, et al. Alcoholism is a disinhibitory disorder: neurophysiological evidence from a Go/No-Go task. Biol Psychol 2005;69(July (3)):353-73.

[55] Burgess PW, Shallice T. Response suppression, initiation and strategy use following frontal lobe lesions. Neuropsychologia 1996;34(April (4)):263-72.

[56] Peelle JE, Cusack R, Henson RNA. Adjusting for global effects in voxel-based morphometry: gray matter decline in normal aging. Neuroimage 2012;60 (April (2)):1503-16.

[57] McClernon FJ. Neuroimaging of nicotine dependence: key findings and application to the study of smoking-mental illness comorbidity. J Dual Diagn 2009;5(April (2)):168-78.
[58] Chye Y, Christensen E, Yücel M. Cannabis use in adolescence: a review of neuroimaging findings. J Dual Diagn 2019;16(July):1-23.

[59] Büchel C, Peters J, Banaschewski T, Bokde ALW, Bromberg U, Conrod PJ, et al. Blunted ventral striatal responses to anticipated rewards foreshadow problematic drug use in novelty-seeking adolescents. Nat Commun 2017;21 (February):8.

[60] Eklund A, Nichols TE, Knutsson H. Cluster failure: why fMRI inferences for spatial extent have inflated false-positive rates. Proc Natl Acad Sci U S A. 2016;113(July (28)):7900-5.

[61] Parvaz MA, Maloney T, Moeller SJ, Malaker P, Konova AB, Alia-Klein N, et al. Multimodal evidence of regional midcingulate gray matter volume underlying conflict monitoring. Neuroimage Clin 2014;5:10-8.

[62] Bush G, Luu P, Posner MI. Cognitive and emotional influences in anterior cingulate cortex. Trends Cogn Sci 2000;4(June (6)):215-22.

[63] Naqvi NH, Rudrauf D, Damasio H, Bechara A. Damage to the insula disrupts addiction to cigarette smoking. Science 2007;315(January (5811)):531-4.

[64] Artiges E, Ricalens E, Berthoz S, Krebs M-O, Penttilä J, Trichard C, et al. IMAGING STUDY: Exposure to smoking cues during an emotion recognition task can modulate limbic fMRI activation in cigarette smokers. Addict Biol 2009;14(4):469-77.

[65] Galandra C, Basso G, Cappa S, Canessa N. The alcoholic brain: neural bases of impaired reward-based decision-making in alcohol use disorders. Neurol Sci 2018;39(March (3)):423-35.

[66] Shidara M, Richmond BJ. Anterior cingulate: single neuronal signals related to degree of reward expectancy. Science 2002;296(May (5573)):1709-11.

[67] Becker A, Gerchen MF, Kirsch M, Hoffmann S, Kiefer F, Kirsch P. Striatal reward sensitivity predicts therapy-related neural changes in alcohol addiction. Eur Arch Psychiatry Clin Neurosci 2018;268(April (3)):231-42.

[68] Dick DM, Smith G, Olausson P, Mitchell SH, Leeman RF, O’Malley SS, et al. Understanding the construct of impulsivity and its relationship to alcohol use disorders. Addict Biol 2010;15(April (2)):217-26.

[69] Schachar RJ, Tannock R, Logan G. Inhibitory control, impulsiveness, and attention deficit hyperactivity disorder. Clin Psychol Rev 1993;13(January (8)):721-39.

[70] Verdejo-García A, Lawrence AJ, Clark L. Impulsivity as a vulnerability marker for substance-use disorders: review of findings from high-risk research, problem gamblers and genetic association studies. Neurosci Biobehav Rev 2008;32(4):777-810. 